\title{
Nahrungsmittelallergien bei Kindern und Jugendlichen
}

Lars Lange

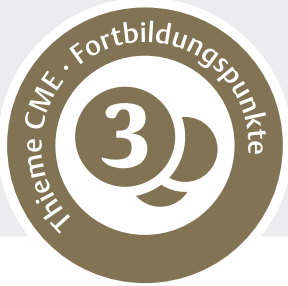

Nahrungsmittelallergien sind häufige Fragestellungen in der pädiatrischen Praxis. Bei genauer Anamnese und diagnostischer Aufarbeitung stellt sich ein Großteil der Vermutungen aber als unbegründet heraus. Die diagnostische Aufarbeitung ist oft einfach, gelegentlich aber auch mühsam, und das gesamte Repertoire der Allergologie von einer gründlichen Anamnese bis hin zu Provokationstestungen ist anzuwenden.

\section{Grundlagen}

Eine Nahrungsmittelallergie ist definiert als eine immunologisch vermittelte Überempfindlichkeitsreaktion, die auf IgE-vermittelten oder zellulären Mechanismen beruhen kann. Damit ist eine klare Abgrenzung gegenüber der Nahrungsmittelintoleranz möglich, die einer nicht immunologisch vermittelten Überempfindlichkeitsreaktion entspricht. Hierbei handelt es sich meist um eine Kohlenhydrat-Malabsorption, bei der die aufgenommenen Kohlenhydrate durch das gastrointestinale Enzymsystem nicht ausreichend aufgespalten werden können (z. B. Laktose- oder Fruktoseintoleranz).

Merke

Anhand des Sensibilisierungsmechanismus unterscheidet man bei den IgE-vermittelten Allergien primäre und sekundäre Nahrungsmittelallergien.

Primäre Nahrungsmittelallergien beruhen auf einer direkten Sensibilisierung gegen das auslösende Nahrungsmittelprotein. Sekundäre Nahrungsmittelallergien basieren auf der Entwicklung von Sensibilisierungen gegen Aeroallergene, die zu einer immunologischen Kreuzreaktion gegen zuvor meist tolerierte Nahrungsmittelproteine führen. Die Grundlage dieser Kreuzreaktionen bilden strukturverwandte (homologe) Proteine, die speziesübergreifend in verschiedenen Pflanzen- und Tierarten enthalten sind.
Entsprechend der Klassifikation in primäre und sekundäre Nahrungsmittelallergien lassen sich sog. Klasse-1- und Klasse-2-Nahrungsmittelallergene voneinander unterscheiden ( Tab. 1). Klasse-1-Nahrungsmittelallergene führen zu primären Nahrungsmittelallergien wie z.B. gegen Hühnerei, Kuhmilch oder Fisch. Klasse-2-Allergene entsprechen typischerweise Nahrungsmitteln, bei denen eine Allergie zumeist über eine Kreuzreaktion entsteht, wie z. B. gegen Apfel, Sellerie oder Pfirsich. Einige Nahrungsmittel können sowohl den Klasse-1- als auch den Klasse-2-Allergenen zugerechnet werden. Typisch ist dies z. B. für Haselnuss, nach deren Verzehr sowohl eine primäre als auch eine sekundäre Nahrungsmittelallergie auftreten kann.

Neben den IgE-vermittelten gibt es noch nicht-IgE-vermittelte Nahrungsmittelallergien. Hier sind die Sensibilisierungswege bei den meisten Erkrankungen noch nicht geklärt. Zu nennen ist hier die allergische Proktokolitis des Säuglings, das Food-Protein-Induced EnterocolitisSyndrom (FPIES) und teilweise auch die eosinophile Ösophagitis.

\section{Epidemiologie}

Nahrungsmittelallergien werden von Patienten häufig vermutet. Circa 16\% (je nach Studie 3-35\%) der Eltern nehmen an, dass ihr Kind unter einer Nahrungsmittelallergie leidet. Erfolgt jedoch eine konsequente allergolo-

- Tab. 1 Einteilung der Nahrungsmittelallergene.

primäre Nahrungsmittelallergene (Kindesalter)

Kuhmilch, Hühnerei, Erdnuss, Schalenfrüchte, Fisch, Weizen, Soja, Saaten (z. B. Sesam) sekundäre Nahrungsmittelallergene

Apfel, Haselnuss, Steinobst, Karotte, Sellerie typischerweise keine Nahrungsmittelallergene

Zucker, Farbstoffe, Konservierungsmittel 
FALLBEISPIEL

\section{Alexander - Teil 1}

Der 7 Monate alte Alexander leidet unter einem atopischen Ekzem. Die Mutter vermutet eine Nahrungsmittelallergie als Auslöser, weil das Ekzem seit der Einführung der Beikost zugenommen hat. Ein PrickTest mit Kuhmilch, Hühnerei, Weizen und Soja ist negativ. Sie erhält die Empfehlung, die Hautpflege zu intensivieren und keine Diät durchzuführen.

6 Wochen später ist das Ekzem gut kontrolliert bei uneingeschränkter Diät.

gische Diagnostik mit dem Endpunkt einer Nahrungsmittelprovokation, ergibt sich eine tatsächliche Prävalenz von nur noch ca. 2\% [1]. Betrachtet man das gesamte Kindes- und Jugendalter in unterschiedlichen Regionen, repräsentiert Kuhmilch das häufigste Nahrungsmittelallergen (0,5-4\%), gefolgt von Erdnuss (0,2-1,9\%), Nüssen $(0,8-1,6 \%)$, Meeresfrüchten inklusive Fisch $(0,2-$ 5,2\%) und Hühnerei (0,2-2\%). Im Gegensatz zur Wahrnehmung der Eltern spielen Intoleranzreaktionen gegen Nahrungsmittelzusatzstoffe wie Konservierungsmittel oder Farbstoffe eine allenfalls untergeordnete Rolle als Auslöser Nahrungsmittel-assoziierter Überempfindlichkeitsreaktionen.

Populationsbezogene epidemiologische Daten zur Nahrungsmittelallergie im Kindes- und Jugendalter liegen nur in begrenztem Umfang vor. Unlängst wurde eine populationsbezogene Studie in Europa veröffentlicht, die aber aufgrund der nur geringen Bereitschaft der Teilnehmer zur Durchführung einer Provokation zur Bestätigung der vermuteten Allergie nur eine große Streubreite bei den Resultaten zwischen 0,8 und 3,8\% angeben konnte [1]. Zusätzlich zeigen diese Ergebnisse ausgeprägte geografische Schwankungen, die auf unterschiedliche Ernährungsgewohnheiten der untersuchten Populationen und andere Umgebungsfaktoren (z. B. Pollenexposition) zurückgehen. So existieren bereits innerhalb Europas signifikante Unterschiede in der Erkrankungshäufigkeit, die ein deutliches „Nord-Süd-Gefälle“ aufweist.

\section{Merke}

Weltweit differieren die Prävalenzraten stark, und Nahrungsmittel, die in unseren Breitengraden als Allergene keine Rolle spielen, können in anderen Regionen häufige Anaphylaxieauslöser darstellen.

Eine weitere Schwierigkeit in der Abschätzung ihrer Prävalenz liegt in der starken Altersabhängigkeit der Nahrungsmittelallergien. Typische Allergien des Kleinkindalters, z.B. gegen Kuhmilch und Hühnerei, werden im späteren Lebensalter weniger relevant, da sich eine hohe
FALLBEISPIEL

Alexander - Teil 2

Alexander knabbert im Alter von 17 Monaten erstmalig an Erdnussflips, die er von seinem Bruder erhalten hat. Er entwickelt rasch eine Lippenschwellung und eine generalisierte Urtikaria. Nachdem er sich einmalig übergeben hat, bessert sich die Situation spontan. Bei der Kinderärztin wird spezifisches IgE für Erdnuss und Ara h 2 bestimmt. Die IgE-Werte liegen bei 4,6 kU/l bzw. 3,8 kU/I. Es wird die Diagnose einer Erdnussallergie gestellt und eine konsequente Meidung von Erdnuss empfohlen. Haselnuss (die er in Form einer Nuss-Nugat-Creme schon zu sich genommen hat) und Cashew-Kerne (die er als Mus schon gegessen hat) soll er weiterhin konsumieren.

Rate an spontaner Toleranzentwicklung einstellen kann. Im Gegensatz hierzu nehmen pollenassoziierte oder andere sekundäre Nahrungsmittelallergien, z.B. Allergien gegen Haselnuss, Apfel oder Krustentiere, mit dem Lebensalter zu. Das bedeutet, dass epidemiologische Untersuchungen in den verschiedenen Altersstufen separat erfolgen müssen.

Bei den nicht-IgE-vermittelten Nahrungsmittelallergien sind ebenfalls keine zuverlässigen Zahlen zur Inzidenz vorhanden. Es ist allerdings bekannt, dass sich ca. die Hälfte der allergischen Reaktionen auf Kuhmilch im 1. Lebensjahr in Form einer nicht-IgE-vermittelten Erkrankung wie der allergischen Proktokolitis oder des FPIES) manifestiert [2]. Die eosinophile Ösophagitis scheint seit ihrer Erstbeschreibung in den 1990er-Jahren mit zunehmender Inzidenz aufzutreten. Allerdings werden in verschiedenen Regionen der Welt stark unterschiedliche Erkrankungszahlen beobachtet, ohne dass es hierfür bislang eine Erklärung gibt.

\section{Ätiologie und Pathogenese}

\section{Primäre lgE-vermittelte Nahrungsmittelallergien}

Die Entstehung einer primären Nahrungsmittelallergie wurde durch zahlreiche Erkenntnisse der letzten Jahre immer besser verstanden. Zentral ist das Prinzip der dualen Allergenexposition bei Säuglingen und Kleinkindern. Hierbei wird davon ausgegangen, dass eine perorale Allergenaufnahme zur Entstehung einer Toleranz führt, wohingegen die epikutane Exposition mit dem allergenen Protein die Allergieentwicklung fördert. So gehen genetische Erkrankungen, die mit einer defekten Hautbarriere assoziiert sind, mit einer deutlich erhöhten Rate an Nahrungsmittelallergien einher. Eine klare Assoziation zeigt 
sich z. B. bei Patienten mit Filaggrin-Defizienz, und zwar unabhängig davon, ob betroffene Kinder eine Hautkrankheit aufweisen oder nicht [3].

\author{
Merke \\ Genetische Erkrankungen, die mit einer defekten \\ Hautbarriere assoziiert sind, gehen mit einer deutlich \\ erhöhten Rate an Nahrungsmittelallergien einher.
}

Überwinden Nahrungsmittelallergene die Hautbarriere, treffen sie auf immunkompetente kutane Zellen, die üblicherweise nicht in Kontakt mit großmolekularen Nahrungsmittelallergenen kommen. Antigenpräsentierende Zellen der Haut wie Langerhans-Zellen prozessieren das Allergen und präsentieren es kutanen T-Lymphozyten, was zur allergenspezifischen Sensibilisierung beitragen kann. Offensichtlich wirkt in diesem Prozess eine vorher schon etablierte orale Toleranz protektiv. Dieser Kontakt zu Nahrungsmittelallergenen kann auf verschiedenen Wegen zustande kommen: Zum einen direkt über den Hautkontakt zwischen Eltern und Kindern, indirekt über allergene Proteine, die im Hausstaub vorhanden sind, oder auch über die Muttermilch. So konnte klar gezeigt werden, dass Muttermilch intakte primäre Proteine der zuvor von der Mutter konsumierten Nahrungsmittel (z. B. Erdnuss, Kuhmilch) enthält. Die Menge der enthaltenen allergenen Proteine ist jedoch von Frau zu Frau unterschiedlich, sodass die Effekte auf Sensibilisierung oder Toleranzentstehung unterschiedlich sein können.

\section{Merke \\ Muttermilch enthält intakte primäre Proteine der zuvor von der Mutter konsumierten Nahrungsmittel und kann so zu einer oralen Toleranz, aber auch Sensibilisierung des Säuglings beitragen.}

Es gibt aber auch Hinweise für eine Allergieentstehung primär über den Gastrointestinaltrakt. Entsprechend deuten bisherige Untersuchungen auf ein erhöhtes Risiko für die Entstehung einer Kuhmilchallergie hin, wenn in den ersten Lebenstagen des Säuglings eine KuhmilchFormula gefüttert, im weiteren Verlauf aber das Kind voll gestillt wird und eine Zufütterung von Kuhmilch unterbleibt.

\section{Merke}

Die intestinale Toleranzentwicklung kann aufgrund einer zu seltenen Exposition ausbleiben, wenn ein Nahrungsmittelallergen nur einmalig konsumiert und dann längere Zeit gemieden wird.

Aktuelle Studien konnten zeigen, dass die frühe Einführung auch potenziell hochallergener Nahrungsmittel wie Erdnuss oder Fisch in die Säuglingsernährung die Rate an Nahrungsmittelallergien senkt [4]. Zahlreiche Untersuchungen deuten darauf hin, dass sich ein optimales Zeitfenster für die Einführung dieser Beikost zwischen dem
4. und 6. Lebensmonat öffnet („window of opportunity“). Dies gilt allerdings nicht für alle Nahrungsmittel. Bei Hühnerei geht eine frühe Einführung von wenig verarbeitetem Protein mit einem erhöhten Allergierisiko einher. Bei vielen Allergenen wie Schalenfrüchten sind keine Daten vorhanden. So sind insgesamt weitere Studien erforderlich, bevor in diesem Zusammenhang differenziertere Handlungsempfehlungen ausgesprochen werden können.

Merke
Säuglingsbeikost sollte nicht zu spät eingeführt
werden und möglichst vielfältig sein. Hochpotente
Allergene sollten besonders bei Risikokindern, also
bei solchen mit mittelschwerem atopischem Ekzem
und/oder Nahrungsmittelallergien, im 1. Lebensjahr
in die Ernährung eingeführt werden, und zwar
besonders dann, wenn das Allergen regelmäßig
im Haushalt konsumiert wird.

\section{Sekundäre IgE-vermittelte Nahrungsmittelallergien}

Sekundäre Allergien gegen Nahrungsmittel beruhen auf Kreuzreaktionen zwischen Nahrungsmittel- und Aeroallergenen, vor allem Pollenallergenen ( $\bullet$ Tab. 2). Hierbei sensibilisiert sich der Patient gegen das pflanzliche oder, in selteneren Fällen, nicht pflanzliche Allergen (z. B. Tropomyosin aus Hausstaubmilben). Im Verlauf der Erkrankung treten dann allergische Reaktionen bei Kontakt mit solchen Nahrungsmitteln auf, die homologe Proteine der gleichen Allergenfamilie enthalten und die Bindung spezifischer lgE-Antikörper ermöglichen. Ob eine sekundäre Nahrungsmittelallergie auftritt, hängt vor allem von der Dauer der Rhinokonjunktivitis und der Höhe des spezifischen IgE ab, weniger von der Schwere der klinischen Symptomatik. In Einzelfällen kann eine sekundäre Allergie auch bestehen, ohne dass zuvor klassische Rhinokonjunktivitis-Beschwerden auftreten.

\begin{abstract}
Merke
Durch gastrale Digestion werden die sekundären pflanzlichen Allergene häufig so weit denaturiert, dass eine IgE-Bindung nicht mehr erfolgen kann. Die meisten sekundären Nahrungsmittelallergene sind thermo- und digestionslabil, sodass sie nur nach Verzehr von rohen Produkten und auch dann nur selten zu systemischen Reaktionen führen.
\end{abstract}

\section{Nicht-IgE-vermittelte zelluläre Nahrungsmittelallergien}

Es ist weitgehend unbekannt, auf welchem Wege spezifische Sensibilisierungen bei nicht-IgE-vermittelten Nahrungsmittelallergien entstehen. Beim FPIES ist auffällig, dass eine besonders frühe Einführung bestimmter Nahrungsmittel wie Hühnerei die Manifestation zu begünstigen scheint. Möglicherweise ist hier ein Zusammenhang mit einer Unreife der gastrointestinalen Barriere oder einer Aktivierung des lokalen Immunsystems zu se- 
- Tab. 2 Klassische Gruppen pollenassoziierter Nahrungsmittelallergene.

\begin{tabular}{|l|l|l|l|}
\hline $\begin{array}{l}\text { zugrunde liegende } \\
\text { Pollensensibilisierung }\end{array}$ & $\begin{array}{l}\text { mögliche sensibilisie- } \\
\text { rende Proteinfamilie }\end{array}$ & Nahrungsmittelallergen & Eigenschaften \\
\hline Birkenpollen & PR-10-Proteine, Profiline & $\begin{array}{l}\text { Haselnuss, Apfel, Sellerie, Karotte, } \\
\text { Pfirsich, Kirsche, Mandel, Erdnuss, Soja }\end{array}$ & $\begin{array}{l}\text { meist thermo- und digestionslabil } \\
\text { (Ausnahme: Gly m4 aus Soja) }\end{array}$ \\
\hline Beifußpollen & $\begin{array}{l}\text { nsLTP, PR-10-Proteine, } \\
\text { Profiline }\end{array}$ & $\begin{array}{l}\text { Sellerie, Gewürze, Paprika, Zwiebel, } \\
\text { Knoblauch, Karotte, Kiwi, Sonnen- } \\
\text { blumensamen, Mango, Kohlsorten, } \\
\text { Petersilie, Senf, Fenchel }\end{array}$ & $\begin{array}{l}\text { bei Beifuß-Sellerie-Assoziation } \\
\text { meist thermo- und digestionsstabil; } \\
\text { bei Beifuß-Birke-Sellerie-Assoziation } \\
\text { meist thermo- und digestionslabil }\end{array}$ \\
\hline Gräserpollen & CCD, Profiline & $\begin{array}{l}\text { Tomate, rohe Kartoffel, Melone, Orange, } \\
\text { Ananas, Gurke, Banane, Aprikose }\end{array}$ & thermo- und digestionslabil \\
\hline
\end{tabular}

hen. Patienten, die an klassischen Erkrankungen dieses Formenkreises leiden (z. B. allergische Säuglings-Proktokolitis, FPIES), weisen keine oder eine nur geringfügig erhöhte Atopierate auf. Es gibt jedoch Fälle, in denen die verschiedenen nicht-IgE- und IgE-vermittelten Erkrankungen ineinander übergehen. Anders stellt sich dies bei der eosinophilen Ösophagitis dar, bei der ein deutlicher Zusammenhang mit einer atopischen Disposition beobachtet wird.

Merke

Nur bei der eosinophilen Ösophagitis wird ein deutlicher Zusammenhang mit einer atopischen Disposition beobachtet.

\section{Auslöser allergischer Reaktionen}

Primäre IgE-vermittelte Nahrungsmittelallergien entstehen meist im 1. Lebensjahr. Typische Auslöser sind Kuhmilch, Hühnerei, Weizen, Erdnuss und Schalenfrüchte. Seltener treten Reaktionen auf Fisch, Sesam oder Hülsenfrüchte wie Soja, Erbsen und Linsen auf. Da Erdnuss und Schalenfrüchte gelegentlich erstmalig im Vorschulalter konsumiert werden, fallen diese Allergien erst dann auf. Ab dem Schulalter werden Allergien gegen Milch, Ei und Weizen seltener, da hier meist die Toleranz eingetreten ist. Stattdessen werden die sekundären Allergien beobachtet. Die einzige Allergenfamilie, bei der regelhaft in jedem Lebensalter eine primäre Allergie auftreten kann, ist Fisch.

\footnotetext{
Merke

Säuglinge können Nahrungsmittelallergene sowohl direkt mit der Säuglingsnahrung als auch über die Muttermilch aufnehmen. Die wichtigsten Allergene sind Kuhmilch, Hühnerei, Weizen und Erdnuss bzw. Nuss. Bei gestillten Kindern sollten daher auch die Ernährungsgewohnheiten der Mütter erfragt werden. Diese können ethnisch unterschiedlich sein, was in der Diagnostik berücksichtigt werden muss.
}

Beim atopischen Ekzem im Säuglings- und Kleinkindalter sind typischerweise Grundnahrungsmittel für eine Ver- stärkung durch Nahrungsmittelallergene verantwortlich. Im Umkehrschluss ist eine Nahrungsmittelallergie jedoch unwahrscheinlich, wenn von den Eltern längere Phasen weitgehender Beschwerdefreiheit bei unveränderter Ernährung beobachtet werden.

Ursache der allergischen Proktokolitis ist mit Abstand am häufigsten die Kuhmilch. Bei der Muttermilch-assoziierten Proktokolitis kann aber selten auch ein anderes, über die Muttermilch übertragenes Allergen relevant sein. Untypisch sind die Auslöser des FPIES. Zwar ist Kuhmilch auch in Deutschland der häufigste Auslöser, aber es kommen auch viele andere ungewöhnliche Allergene wie Fleisch, Meeresfrüchte, Gemüse wie Kartoffel und Kürbis oder Getreide wie Hafer und Weizen und Reis in Betracht.

\section{Klinik}

Das Spektrum klinischer Symptome ist bei Nahrungsmittelallergien besonders groß, nicht zuletzt aufgrund der verschiedenen Erkrankungen. Im Folgenden werden die unterschiedlichen klinischen Bilder vorgestellt.

\section{IgE-vermittelte Soforttyp-Reaktionen}

Primäre Nahrungsmittelallergien

Primäre Nahrungsmittelallergien führen zu den typischen Symptomen einer systemischen allergischen SoforttypReaktion bis hin zum Vollbild der Anaphylaxie [5]. Nach dem zeitlichen Ablauf der Reaktion wird in schnelle, innerhalb von Sekunden bis zu 60 Minuten auftretende und sich später manifestierende Reaktionen unterschieden. Bei verzögerter Resorption des Allergens kann der Beginn der allergischen Reaktion erst bis zu 2 Stunden nach Ingestion eintreten.

Merke

In der Regel zeigen Soforttyp-Reaktionen eine Spontanremission innerhalb eines Tages. Dies hilft häufig bei der Differenzierung zwischen einer Urtikaria anderer Ursache (z. B. infektassoziiert) und einer Nahrungsmittelallergie. 
Das häufigste Manifestationsorgan ist die Haut. Sie zeigt oft eine Kontakturtikaria im Bereich der unmittelbaren Berührung durch das auslösende Allergen, zumeist in der Perioralregion. Bei Säuglingen und Kleinkindern kann sie isoliert bestehen. Dies bedeutet, dass trotz eindeutiger Kontakturtikaria eine Verträglichkeit bei oraler Nahrungsaufnahme möglich ist. Weitere Symptome umfassen eine generalisierte Urtikaria und/oder Angioödeme.

Bronchopulmonale Soforttyp-Symptome entsprechen denen bei Anaphylaxie. Sie reichen von Beschwerden im Bereich der oberen bis zu den tiefen Atemwegen. Es sind jedoch jeweils nur Akutsymptome vorhanden.

\footnotetext{
Merke

Wiederholte, obstruktive Bronchitiden oder dauerhafte respiratorische Symptome sind keine typischen Folgen einer Nahrungsmittelallergie.
}

In gleicher Weise äußert sich eine gastrointestinale Beteiligung vor allem in akut auftretender Übelkeit, Erbrechen, Durchfall und Bauchschmerzen. Untypisch sind chronische Bauchschmerzen als Folge einer Nahrungsmittelallergie. Der Zusammenhang zwischen Aufnahme des Allergens und Symptomen ist in aller Regel klar herstellbar und damit nur episodisch vorhanden. Die Beschwerden sind nur selten ausschließlich auf den Gastrointestinaltrakt begrenzt, sondern eher begleitet von Symptomen an weiteren Organsystemen, besonders der Haut.

Besonders bei Jugendlichen ist auch eine Beteiligung des Kreislaufs möglich. Diese zeigen sich durch Tachykardie, Hypotonie, Blässe bis hin zum Kollaps.

\section{Pollenassoziierte sekundäre Nahrungsmittelallergien}

Pollenassoziierte Nahrungsmittelallergien (PAN) führen typischerweise zu einem oralen Allergiesyndrom (OAS). Hierbei verspüren die Patienten rasch nach Aufnahme enorale und pharyngeale Parästhesien, die häufig als Kribbeln oder Brennen der Schleimhaut beschrieben werden. Zusätzlich kann es zu Ödemen von Lippen, Zunge oder Gaumen kommen. Wenn das Schleimhautödem den unteren Rachenraum betrifft, können sich Dyspnoe und eine obere Atemwegsobstruktion einstellen. Klinisch relevante Befunde im Sinne eines Stridors bis hin zur Hypoxie sind jedoch selten. In sehr ausgeprägten Fällen treten auch systemische Beschwerden auf. Am häufigsten ist eine generalisierte Urtikaria zu beobachten, aber auch gastrointestinale Symptome und eine Obstruktion der tiefen Atemwege sind beschrieben [5].

\footnotetext{
Merke

Die Symptomatik bei PAN unterliegt in Schwere und Ausprägung ausgeprägten saisonalen Schwankungen. Häufig ist sie in oder kurz nach der Pollensaison stark ausgeprägt, während die Beschwerden unmittelbar vor der nächsten Pollensaison am mildesten sind.
}

\section{Zellulär vermittelte gastrointestinale Nahrungsmittelallergien}

Allergische Proktokolitis

Allergische Proktokolitiden des Säuglings können sich bereits in den ersten Lebenstagen manifestieren, in denen sich sowohl unter ausschließlicher Muttermilchernährung als auch nach Beginn der Kuhmilch-Beifütterung entsprechende Beschwerden entwickeln. Sie äußert sich in blutigen Stühlen, typischerweise erkennt man Blutfäden in der Windel. Es können kolikartige Bauchschmerzen auftreten, aber meist ist der Allgemeinzustand der Kinder nicht beeinträchtigt. Nur selten sind die beobachteten Blutungen Ursache einer Anämie. Die Beschwerden sind bei gestillten Kindern oft geringer ausgeprägt als bei Formula-ernährten.

\section{Food-Protein-Induced Enterocolitis-Syndrom (FPIES)}

Das FPIES ist eine zunehmend erkannte Erkrankung, die unterschiedliche klinische Verlaufsformen hat und in jedem Lebensalter auftreten kann. Man unterscheidet die chronische Verlaufsform, die nur bei jungen Säuglingen in den ersten Lebensmonaten beobachtet wird, und die akute, die auch bei Erwachsenen auftritt [6]. Die chronische Verlaufsform zeichnet sich durch ein oft schweres Krankheitsbild aus, das mit einer Gedeihstörung und Erbrechen einhergeht. Teilweise geht es so weit, dass die Kinder unter dem Bild einer Sepsis mit schwerer Dehydratation und Stoffwechselentgleisungen stationär intensivmedizinisch behandelt werden. Die Voraussetzung für die chronische Verlaufsform ist eine regelmäßige tägliche Aufnahme eines Allergens, in der Regel die Formulanahrung des Kindes auf Milchbasis. Leichtere Formen treten bei Muttermilchernährung auf, wenn das Kind nur mit geringen Allergenmengen konfrontiert ist.

\section{FALLBEISPIEL}

Anna

Die 8 Monate alte Anna wird zum 2. Mal innerhalb von 6 Wochen mit schwerem, akut aufgetretenem Erbrechen stationär aufgenommen. Bei Aufnahme ist sie blass und apathisch. Aufgrund des stark eingeschränkten Allgemeinzustands erfolgt eine intravenöse Rehydratation. Nach 24 Stunden ist sie jeweils wieder gesund. Die Erregerdiagnostik bleibt negativ. Der Mutter fällt auf, dass Anna jedes Mal ca. 2 Stunden vorher ein Gläschen mit Lachs konsumiert hat. Alle anderen Zutaten des Gläschens vertrug sie zwischen den Ereignissen symptomlos. Das spezifische lgE für Lachs ist negativ. Aufgrund der eindeutigen Anamnese mit wiederholtem, verzögert auftretendem Erbrechen nach Lachskonsum ohne Symptome einer allergischen Sofortreaktion wird der Verdacht auf ein akutes FPIES geäußert. Anna erfüllt das Majorkriterium und 5 Minorkriterien ( $\vee$ Tab. 3), sodass die Diagnose gestellt werden kann. Der Mutter wird eine Meidung von Fisch und eine Reevaluation nach ca. 2-3 Jahren empfohlen. 
- Tab. 3 Diagnosekriterien des akuten und chronischen FPIES [6].

\section{akute FPIES}

- Majorkriterium: Erbrechen 1-4 h nach Ingestion des verdächtigten Nahrungsmittels ohne Zeichen einer IgE-vermittelten Reaktion wie Haut- oder respiratorischen Beschwerden

- Minorkriterien (3 oder mehr):

- eine 2. Episode von Erbrechen nach Aufnahme desselben Nahrungsmittels

- wiederholtes Erbrechen 1-4 h nach Aufnahme eines anderen Nahrungsmittels

- extreme Lethargie in Zusammenhang mit der Reaktion

- deutliche Blässe in Zusammenhang mit der Reaktion

- Notwendigkeit einer Notfallbehandlung in Zusammenhang mit einer Reaktion

- Notwendigkeit der intravenösen Flüssigkeitssubstitution in Zusammenhang mit einer Reaktion

- Durchfall innerhalb von $24 \mathrm{~h}$ (meist 5-10 h)

- Hypotension

- Hypothermie

\section{chronische FPIES}

- schweres Erscheinungsbild: bei regelmäßigem Konsum (z. B. Formulanahrung) intermittierendes, aber zunehmendes Erbrechen und teils blutiger Durchfall, gelegentlich mit Dehydratation und Azidose

- mildes Krankheitsbild: bei Konsum geringerer Dosen (z. B. Allergenzufuhr über die Muttermilch) intermittierendes Erbrechen und/oder Durchfall mit Gedeihstörung ohne Dehydratation oder Azidose

- Das wichtigste Kriterium ist das Verschwinden der Symptome innerhalb weniger Tage bei Diät und Auftreten akuter FPIES-Symptome bei Wiedereinführung.

- Ohne Provokation ist die Diagnose nicht sicher zu stellen.
Kommt es nur gelegentlich zu einem Allergenkontakt, wird die akute Verlaufsform beobachtet. Diese tritt auch auf, wenn ein Kind mit chronischer FPIES nach einer Karenzphase erneut exponiert wird. Kennzeichnend ist ein schweres Erbrechen, welches mit einer meist für den Patienten und sein Allergen festen Latenz von 1-4 Stunden auftritt. Die akute Symptomatik ist ebenfalls so schwer, dass oft eine stationäre Betreuung mit parenteraler Flüssigkeitszufuhr erforderlich wird. Da es keine typischen wegweisenden Laborparameter gibt, basiert die Diagnose auf der Anamnese. Die typischen Diagnosekriterien finden sich in > Tab. 3.

\section{Eosinophile Ösophagitis}

Die eosinophile Ösophagitis wird vermutlich bei einem Großteil der Patienten ebenfalls durch Nahrungsmittelallergene ausgelöst oder zumindest verstärkt. Ihre Symptomatik ist altersabhängig. Bei stabilem bis gutem Allgemeinzustand fallen betroffene Säuglinge und Kleinkinder aufgrund von Erbrechen, Irritabilität oder Bauchschmerzen auf. Im späteren Kindesalter werden zusätzlich Übelkeit, Regurgitationen, Dysphagien oder retrosternales Brennen geschildert. Endoskopisch lässt sich eine Ösophagitis mit deutlicher Vermehrung der intraepithelialen Eosinophilen nachweisen.

Betroffene Patienten essen oft auffällig langsam und kauen sehr gründlich. Bereits bei Kindern kann die häufiger bei Jugendlichen und Erwachsenen beobachtete Nahrungsmittel-Impaktion auftreten. Hierunter versteht man ein Bolus-Ereignis, bei dem nicht ausreichend gekaute Nahrung (v.a. Fleisch, Brot) im Ösophagus „steckenbleibt" und akute Schmerzen verursacht. Diese Impaktionen sind häufig so hartnäckig, dass eine endoskopische Entfernung des Speisebolus notwendig wird.

\section{Nahrungsmittelallergien bei atopischem Ekzem}

Bei Kindern und Jugendlichen mit atopischem Ekzem können sowohl isolierte Sofort- und Spättyp-Reaktionen als auch eine Kombination aus beiden beobachtet werden. Hierbei können Exazerbationen des atopischen Ekzems schon wenige Stunden nach Ingestion eines Nahrungsmittelallergens oder aber sehr selten erst nach einer erheblich längeren Latenz von bis zu 72 Stunden auftreten. Diese verzögerten Reaktionen werden häufig von Eltern vermutet, in Provokationstestungen lassen sie sich aber kaum nachweisen, sodass mittlerweile davon ausgegangen wird, dass eine isolierte Spätreaktion ohne initiale Sofortreaktion wie zumindest eine periorale Urtikaria oder ein Flush unmittelbar nach Aufnahme des Nahrungsmittels selten ist.

\footnotetext{
Merke

Eine Nahrungsmittelallergie als relevanter Auslöser sollte nur dann vermutet werden, wenn das Ekzem mindestens mittelschwer ist und eine adäquat durchgeführte Therapie keine anhaltende Stabilisierung des Befundes ergibt.
}

Während die Nahrungsmittelallergie im Säuglingsalter einen relevanten Triggerfaktor darstellt, ist ein Ekzem bei größeren Kindern jenseits des 2. Lebensjahrs nur noch in Ausnahmefällen durch eine Nahrungsmittelallergie beeinflusst. Auch bei Ekzemen, die erst nach dem 1. Lebensjahr beginnen, spielt eine Nahrungsmittelallergie in der Regel eine untergeordnete Rolle.

Es ist zu bedenken, dass Kinder mit einem atopischen Ekzem oft das Risiko haben, unbemerkt gegen potente Allergene sensibilisiert zu sein. Um Anaphylaxien bei Erstkontakt zu vermeiden, ist es sinnvoll, Sensibilisierungen 
- Tab. 4 Auslöser und sinnvolle Diagnostik bei atopischem Ekzem im Kindes- und Jugendalter [7].

\begin{tabular}{|c|c|c|c|c|c|c|}
\hline $\begin{array}{l}\text { Alter bei } \\
\text { Diagnostik }\end{array}$ & $\begin{array}{l}\text { bis } 6 \text {. Lebens- } \\
\text { monat, nicht/ } \\
\text { teilgestillt }\end{array}$ & $\begin{array}{l}\text { bis } 6 . \text { Lebens- } \\
\text { monat, voll- } \\
\text { gestillt }\end{array}$ & $\begin{array}{l}\text { 6.-18. Lebens- } \\
\text { monat }\end{array}$ & $\begin{array}{l}\text { 18.-36. Lebens- } \\
\text { monat }\end{array}$ & Vorschulkinder & $\begin{array}{l}\text { Schulkinder und } \\
\text { Jugendliche }\end{array}$ \\
\hline \multicolumn{7}{|l|}{ leichtes Ekzem } \\
\hline präventive Diagnostik & \multicolumn{4}{|c|}{$\begin{array}{l}\text { keine bei fehlender Sofortreaktion; bzw. bei gegebener Indikation präventive Diag- } \\
\text { nostik (abhängig vom elterlichen Konsum): Ei, Erdnuss, Haselnuss, Cashew, Walnuss }\end{array}$} & \multicolumn{2}{|c|}{$\begin{array}{l}\text { keine bei fehlender Sofortreaktion und } \\
\text { fehlenden anamnestischen Hinweisen } \\
\text { auf Saisonalität bzw. häusliche Exposi- } \\
\text { tion Gräser-, Birkenpollen, Tiere, Haus- } \\
\text { staubmilben }\end{array}$} \\
\hline \multicolumn{7}{|c|}{ mittelschweres - schweres Ekzem } \\
\hline $\begin{array}{l}\text { Triggerfaktoren des } \\
\text { chronischen Ekzems } \\
\text { (abhängig von Konsum, } \\
\text { bzw. Exposition) }\end{array}$ & Kuhmilch & $\begin{array}{l}\text { Kuhmilch, Wei- } \\
\text { zen, Hühnerei, } \\
\text { Erdnuss, Hasel- } \\
\text { nuss; Weiteres } \\
\text { nach mütter- } \\
\text { lichem Konsum }\end{array}$ & $\begin{array}{l}\text { Kuhmilch, Wei- } \\
\text { zen, Hühnerei, } \\
\text { Soja, Hausstaub- } \\
\text { milbe, Tierepithe- } \\
\text { lien }\end{array}$ & $\begin{array}{l}\text { Hausstaubmilbe, } \\
\text { Katzen-, Hunde- } \\
\text { epithelien }\end{array}$ & \multicolumn{2}{|c|}{$\begin{array}{l}\text { Pollen nach Saisonalität, Hausstaub- } \\
\text { milbe, Katzen-, Hundeepithelien }\end{array}$} \\
\hline $\begin{array}{l}\text { präventive Diagnostik } \\
\text { (abhängig von häus- } \\
\text { licher Exposition und } \\
\text { Konsum des Kindes) }\end{array}$ & \multicolumn{2}{|c|}{$\begin{array}{l}\text { Hühnerei, Erdnuss, Haselnuss, Cashew, } \\
\text { Walnuss, Sesam }\end{array}$} & $\begin{array}{l}\text { Hühnerei, Erd- } \\
\text { nuss, Haselnuss, } \\
\text { Cashew, Walnuss, } \\
\text { Sesam }\end{array}$ & $\begin{array}{l}\text { Erdnuss, Hasel- } \\
\text { nuss, Cashew, } \\
\text { Walnuss, Sesam }\end{array}$ & $\begin{array}{l}\text { Erdnuss, Hasel- } \\
\text { nuss, Cashew, } \\
\text { Walnuss }\end{array}$ & $\begin{array}{l}\text { nur gezielt bei si- } \\
\text { cher fehlendem } \\
\text { Konsum potenter } \\
\text { Allergene! }\end{array}$ \\
\hline
\end{tabular}

auf bislang nicht konsumierte, potente Nahrungsmittel zu überprüfen (präventive Diagnostik, > Tab. 4) [7].

\section{Diagnostik}

\section{Anamnese}

Der Anamnese kommt zur Planung der weiteren Diagnostik wie in allen Bereichen der Allergologie eine entscheidende Bedeutung zu. Zunächst ist festzuhalten, welche Symptome nach Verzehr welcher Nahrungsmittel und in welchem zeitlichen Ablauf aufgetreten sind. Da die Entwicklung einer zwischenzeitlichen Toleranz möglich ist, müssen zusätzlich der Zeitpunkt des letztmaligen Verzehrs des vermuteten Auslösers und die entsprechende Reaktion erfragt werden. Zur Analyse der infrage kommenden Allergene sollte nach einer Reaktion die möglichst exakte Zusammensetzung der auslösenden Mahlzeit dokumentiert werden. Bei Fertigprodukten kann hierfür die Zutatenliste auf der Verpackung herangezogen werden. Des Weiteren sollten die Ernährungsgewohnheiten des Patienten und seiner Familie dokumentiert werden. Zu erfragen ist, welche Nahrungsmittel regelmäßig oder bisher überhaupt nicht konsumiert bzw. bewusst gemieden wurden. Eine Beratung durch eine qualifizierte Ernährungsfachkraft und/oder das Führen eines Ernährungstagebuchs sind hierbei schon im Vorfeld eine große Hilfe.

\section{Hauttestung}

Als In-vivo-Testmethode der Wahl erfolgt ein Haut-PrickTest mit kommerziell erhältlichen Testlösungen oder mit nativen Nahrungsmitteln in Prick-zu-Prick-Technik. Einige
Nahrungsmittel enthalten biogene Amine und andere irritative Substanzen, die zu einem hohen Prozentsatz falsch positiver Befunde führen und daher für die Hauttestung ungeeignet sind ( $\triangleright$ Tab.5). Zahlreiche Untersuchungen deuten darauf hin, dass die diagnostische Sensitivität des Haut-Prick-Tests mit der Sensitivität der serologischen IgE-Diagnostik vergleichbar ist.

\section{Merke \\ Bei positiver Testreaktion sollte gegenüber Eltern und Patienten deutlich darauf hingewiesen werden, dass diese zunächst nur eine Sensibilisierung („Mög- lichkeit einer Allergie“) anzeigt, die ggf. mittels ora- ler Provokationstestung objektiviert werden muss.}

Auch eine Korrelation des Quaddeldurchmessers mit der Wahrscheinlichkeit oder Ausprägung einer klinischen Symptomatik ist nicht zuverlässig möglich. Der früher gelegentlich empfohlene Atopie-Patch-Test hat heute keine Bedeutung mehr. Es gibt eine hohe Zahl an falsch positiven und negativen Befunden. Somit ist der diagnostische Zusatznutzen sehr begrenzt.

\section{Labordiagnostik}

\section{IgE-Serologie}

Bei der Bestimmung spezifischer IgE-Antikörper muss bedacht werden, dass ca. $20 \%$ der deutschen Kinder mindestens eine IgE-vermittelte Sensibilisierung gegen Nahrungsmittel aufweisen, während nur wenige dieser Kinder wirklich an einer Nahrungsmittelallergie erkrankt sind. Somit sollten - wie auch nach positiver Prick-Testung - Eltern und Patienten auf die häufig fehlende klinische Relevanz positiver IgE-Screenings hingewiesen werden. 
- Tab. 5 Eignung verschiedener, pädiatrisch relevanter Nahrungsmittel für den Haut-Prick-Test [6].

\begin{tabular}{|c|c|c|c|}
\hline & kommerzieller Extrakt & Nativtestung geeignet & $\begin{array}{l}\text { Nativtestung bedingt } \\
\text { geeignet }\end{array}$ \\
\hline Fisch & $x$ & $x$ & - \\
\hline Schalentiere & $x$ & $\mathrm{x}$ & - \\
\hline Hühnerei & $x$ & $x$ & - \\
\hline Milch & $x$ & $\mathrm{x}$ & - \\
\hline Erdnuss/Schalenfrüchte & $x$ & $x$ & - \\
\hline Ölsaaten (Sesam, Mohn etc.) & - & $x$ & - \\
\hline Weizen/Zerealien & $(x)$ & $x$ & - \\
\hline Kernobst (Apfel etc.) & - & $x$ & - \\
\hline Steinobst (Pfirsich etc.) & $(x)$ & $x$ & - \\
\hline Soja & $(x)$ & $x$ & - \\
\hline Erdbeere & - & - & $x$ \\
\hline Tomate & - & - & $x$ \\
\hline Kiwi & - & - & $x$ \\
\hline Sellerie & $(x)$ & $x$ & - \\
\hline Gewürze & - & - & $x$ \\
\hline Senf & - & - & $x$ \\
\hline
\end{tabular}

Es wurde lange Zeit angenommen, dass aus der Höhe allergenspezifischer lgE-Titer auf das Risiko einer klinischen Reaktion geschlossen werden kann. Dies ist aber so für die Mehrzahl der Kinder nicht anwendbar. Auch die Schwere der allergischen Reaktion oder die individuelle Schwellendosis sind nicht von der Höhe des spezifischen IgE abzulesen. Daher lassen sich im klinischen Alltag immer wieder Patienten beobachten, die trotz sehr niedriger IgE-Titer schwere Anaphylaxien gegen Nahrungsmittel entwickeln oder aber bei sehr hohen spezifischen IgE-Titern keinerlei klinische Reaktionen zeigen.

Merke

Für die Mehrzahl der Kinder kann aus der Höhe allergenspezifischer IgE-Titer nicht auf das Risiko einer klinischen Reaktion geschlossen werden.

Seit einigen Jahren ist es mithilfe der sog. komponentenbasierten Diagnostik möglich, spezifische lgE-Antikörper gegen Einzelmoleküle zahlreicher Allergenquellen zu bestimmen. Anders als in der Diagnostik mit Allergenextrakten lassen sich auf diese Art Soforttyp-Sensibilisierungen gegen einzelne Nahrungsmittelproteine (Allergenkomponenten) untersuchen ( $\triangleright$ Tab. 6). Die Kenntnis des auslösenden allergenen Proteins kann in der Diagnostik von Nahrungsmittelallergien hilfreich sein, z. B. um Kreuzsensibilisierungen gegen Proteine einer Allergenfamilie nachzuweisen.
Am besten untersucht ist die komponentenbasierte Diagnostik bei Verdacht auf eine Allergie gegen Erdnuss. So konnten viele Studien zeigen, dass der Nachweis spezifischer IgE-Antikörper gegen das Erdnuss-Speicherprotein Ara h 2 mit einem hohen Risiko systemischer Reaktionen assoziiert ist $[8,9]$. Dieses Protein gehört zur Familie der Speicherproteine. Neben Ara h 2 aus Erdnuss konnte auch für Ana o 3 aus Cashew, Cor a 14 und Cor a 9 aus Haselnuss und Jug $r 1$ aus der Walnuss gezeigt werden, dass sie gute Markerproteine sind, um das Risiko einer anaphylaktischen Reaktion nach Konsum der Nahrungsmittel anzuzeigen, und zwar genauer als das slgE gegen den Gesamtextrakt [9-11].

Eine signifikante Erhöhung des IgE-Werts gegen einen der genannten Risikomarker ist daher in vielen Situationen zusammen mit der Anamnese ein ausreichend gutes Kriterium für die Diagnose einer primären Allergie und kann so die Durchführung einer Provokation mit dem jeweiligen Nahrungsmittel ersparen. Es muss aber bedacht werden, dass es auch Patienten mit hohem spezifischem IgE-Spiegel gegen ein Speicherprotein gibt, die nicht allergisch sind, und Patienten ohne eine solche Sensibilisierung, die doch allergisch sind.

Bei Verdacht auf eine pollenassoziierte Nahrungsmittelallergie (PAN) ist die komponentenbasierte Diagnostik nur begrenzt hilfreich. So zeigt sich aufgrund einer ausgeprägten Homologie relevanter Pollen- und Nahrungsmittelallergene (z.B. PR-10-Proteine bei Birkenpollen- 
- Tab. 6 Auswahl potenziell hilfreicher Allergenkomponenten zur serologischen Diagnostik IgE-vermittelter Sensibilisierungen gegen Nahrungsmittelallergene [8].

\begin{tabular}{|c|c|c|c|c|}
\hline $\begin{array}{l}\text { Name des } \\
\text { Allergens }\end{array}$ & Allergenfamilie & Quelle & chemische Eigenschaften & klinische Relevanz \\
\hline Pru p 1 & PR 10-Protein & \multirow{2}{*}{$\begin{array}{l}\text { Pfirsich, große } \\
\text { Homologie zu ande- } \\
\text { ren Obstsorten }\end{array}$} & thermolabil, digestionslabil & klassisches OAS bei PAN \\
\hline Pru p 3 & nsLTP & & $\begin{array}{l}\text { thermostabil, } \\
\text { digestionsstabil }\end{array}$ & Risiko einer Anaphylaxie (selten in Mitteleuropa) \\
\hline Gly m 4 & PR 10-Protein & Soja & $\begin{array}{l}\text { eingeschränkt thermolabil } \\
\text { und digestionslabil }\end{array}$ & Risiko einer Anaphylaxie bei Konsum großer Mengen \\
\hline Ara h 8 & PR 10-Protein & Erdnuss & $\begin{array}{l}\text { thermolabil, } \\
\text { digestionslabil }\end{array}$ & $\begin{array}{l}\text { kaum klinische Relevanz bei gleichzeitigem Fehlen } \\
\text { einer Speicherproteinsensibilisierung }\end{array}$ \\
\hline Ara h 2, Ara h 6 & $\begin{array}{l}\text { Speicherprotein, } \\
\text { 2S-Albumin }\end{array}$ & Erdnuss & $\begin{array}{l}\text { thermostabil, } \\
\text { digestionsstabil }\end{array}$ & $\begin{array}{l}\text { hohe Vorhersagekraft einer systemischen allergi- } \\
\text { schen Reaktion (meist nur Ara h } 2 \text { erforderlich) }\end{array}$ \\
\hline Cor a 1 & PR 10-Protein & Haselnuss & $\begin{array}{l}\text { thermolabil, } \\
\text { digestionslabil }\end{array}$ & $\begin{array}{l}\text { klassisches OAS bei gleichzeitigem Fehlen einer } \\
\text { Speicherproteinsensibilisierung }\end{array}$ \\
\hline Cor a 9, Cor a 14 & Speicherproteine & Haselnuss & $\begin{array}{l}\text { thermostabil, } \\
\text { digestionsstabil }\end{array}$ & $\begin{array}{l}\text { hohe Vorhersagekraft einer systemischen } \\
\text { allergischen Reaktion (besonders Cor a 14) }\end{array}$ \\
\hline Ana o 3 & $\begin{array}{l}\text { Speicherprotein, } \\
\text { 2S-Albumin }\end{array}$ & Cashew & $\begin{array}{l}\text { thermostabil, } \\
\text { digestionsstabil }\end{array}$ & $\begin{array}{l}\text { hohe Vorhersagekraft einer systemischen } \\
\text { allergischen Reaktion }\end{array}$ \\
\hline Jug r 1 & $\begin{array}{l}\text { Speicherprotein, } \\
\text { 2S-Albumin }\end{array}$ & Walnuss & $\begin{array}{l}\text { thermostabil, } \\
\text { digestionsstabil }\end{array}$ & $\begin{array}{l}\text { hohe Vorhersagekraft einer systemischen } \\
\text { allergischen Reaktion bei Kindern }\end{array}$ \\
\hline Jug r 3 & nsLTP & Walnuss & $\begin{array}{l}\text { thermostabil, } \\
\text { digestionsstabil }\end{array}$ & Risiko einer Anaphylaxie (selten in Mitteleuropa) \\
\hline Gad c 1, Cyp p 1 & Parvalbumin & Dorsch, Karpfen & $\begin{array}{l}\text { thermostabil, } \\
\text { digestionsstabil }\end{array}$ & $\begin{array}{l}\text { Kreuzreaktion zu anderen Fischen mit weißem } \\
\text { Muskelfleisch }\end{array}$ \\
\hline
\end{tabular}

\section{FALLBEISPIEL}

\section{Alexander-Teil 3}

Im Alter von 5 Jahren klagt Alexander nach dem Knacken und Verzehr einer frischen Haselnuss aus dem Garten über Kribbeln im Mund, und die Lippe schwillt an. Die Mutter verabreicht sofort Cetirizin aus dem Notfallset. Weitere Beschwerden bestehen nicht. Alexander hatte im Frühjahr erstmals Zeichen eines Heuschnupfens entwickelt. Nuss-Nugat-Creme kann er weiter ohne Symptome essen. Bei einer erneuten Laboruntersuchung zeigen sich folgende IgE-Werte: Birke: 34,7 kU/l; Haselnuss: 28,1 kU/l; Cor a 1: 31,6 kU/l; Cor a 14 und Cor a 9: negativ; Ara h 2: $0,8 \mathrm{kU} / \mathrm{l}$. Es wird die Diagnose einer Birkenpollenassoziierten Haselnussallergie gestellt und vom Verzehr ungerösteter Haselnüsse abgeraten.

allergie) häufig eine serologische Kreuzreaktivität zu den getesteten Nahrungsmitteln, die nicht regelhaft mit einem oralen Allergiesyndrom assoziiert ist.

\begin{abstract}
Merke
Im Praxisalltag kann es zunächst sinnvoll sein, nach spezifischem IgE gegen die Leitallergene der auslösenden Allergenfamilien zu fahnden: Bet v 1 aus der Birke für die PR 10-Proteine und Phl p 12 aus Lieschgras für die Profiline.
\end{abstract}

Zum Ausschluss einer systemischen Reaktion bei Verdacht auf eine Obst- oder Gemüseallergie ist es ebenfalls sinnvoll, IgE-Antikörper gegen das nichtspezifische LipidTransfer-Protein (nsLTP) aus Pfirsich (Pru p 3) zu analysieren. Es ist bekannt, dass Patienten mit einer Allergie gegen diese Proteinfamilie zu anaphylaktischen Reaktionen neigen. Man findet diese Form der Obstallergie vornehmlich bei Patienten aus dem Mittelmeerraum.

\section{Laboruntersuchungen bei \\ nicht-IgE-vermittelten Erkrankungen}

Bei den nicht-IgE-vermittelten Nahrungsmittelallergien sind, wie der Name nahelegt, meist keine spezifischen IgE-Antikörper im Serum nachweisbar. Als Marker für die Aktivität der Erkrankung kann die Eosinophilenzahl im Blut oder das eosinophile kationische Protein (ECP) dienen. Im Gegensatz dazu sind akute Laborveränderungen beim FPIES regelhaft vorhanden. Die Kinder zeigen im Rahmen der akuten Entzündungsreaktion eine periphere Neutro-/Eosinophilie und eine Thrombozytose. Durch Malabsorption und Dehydratation entsteht eine Azidose, 
die mit transienten Methämoglobinämien verbunden sein kann. Spezifisches lgE gegen das auslösende Allergen entwickelt nur ein geringer Teil der Kinder. Inwieweit dies klinische Bedeutung hat, ist unklar [6].

Weitere Methoden der Labordiagnostik, insbesondere zelluläre Tests, lassen keinen zusätzlichen Informationsgewinn erwarten. Gänzlich abzulehnen ist eine lgG-basierte Diagnostik, bei der spezifische lgG- und IgG4-Antikörper gegen viele Nahrungsmittelallergene gemessen werden, um daraus Ernährungsempfehlungen abzuleiten. Dadurch, dass die IgG-Synthese eine physiologische Reaktion des Körpers auf Fremdantigene darstellt, finden sich regelhaft zahlreiche falsch positive Testergebnisse. Daher und wegen der Gefahr völlig unnötiger, potenziell gefährlicher Karenzdiäten wird in aktuellen Positionspapieren nationaler und internationaler Fachgesellschaften von der Bestimmung nahrungsmittelspezifischer IgG-Antikörper in der klinischen Diagnostik abgeraten [12].

\section{Orale Provokationstestungen}

Die orale Provokationstestung stellt den diagnostischen Goldstandard bei Verdacht auf eine Nahrungsmittelallergie dar. Ihre Durchführung ist jedoch aufwendig und meist mit einem voll- oder teilstationären Aufenthalt verbunden. Um den entsprechenden Aufwand zu rechtfertigen, sollte die Provokationstestung daher stets mit dem Ziel unternommen werden, den Verdacht auf eine Nahrungsmittelallergie eindeutig zu bestätigen oder zu entkräften. Provokationen können offen oder doppelblind unternommen werden ( $\triangleright$ Tab. 7). Die Durchführung von Provokationstestungen wurde unlängst in einem Manual von der wissenschaftlichen Arbeitsgemeinschaft der GPA detailliert beschrieben [13].
Vor der Provokation muss das zu testende Nahrungsmittel gemieden werden. Bei allergischen Sofortreaktionen ist dies obligat der Fall, bei Kindern mit atopischem Ekzem hingegen sollte vor einer Provokation zunächst durch eine Besserung unter Diät die potenzielle Relevanz einer Sensibilisierung bestätigt werden. Es ist zu beachten, dass unter einer längeren und konsequenten Eliminationsdiät eine Änderung des Reaktionsmusters auftreten kann. So kann es im Verlauf zu IgE-vermittelten allergischen Soforttyp-Reaktionen kommen, auch wenn zuvor ausschließlich nahrungsmittelassoziierte Ekzem-Exazerbationen beobachtet wurden [15].

\begin{abstract}
Merke
Die Eliminationszeit sollte nicht zu lange ausgedehnt werden, auch wenn exakte Grenzwerte bisher nicht definiert sind. Bei einer Eliminationsdauer $>4$ Wochen steigt das Risiko einer Sofortreaktion mit hoher Wahrscheinlichkeit an, sodass eine Re-Exposition dann nur noch unter adäquatem Monitoring erfolgen sollte.
\end{abstract}

Bei Kindern mit nicht-IgE-vermittelter Nahrungsmittelallergie sollten die Beschwerden im Rahmen der Eliminationsdiät möglichst vollständig kontrolliert sein, bevor eine Provokationstestung erfolgt. Bei Säuglingen mit allergischer Proktokolitis ist bei milden Verläufen eine häusliche Re-Exposition möglich. Bei FPIES drohen bei der Provokation schwere akute Reaktionen. Hier ist eine stationäre Überwachung obligat.

Nach jeder Provokationstestung ist ein ausführliches Beratungsgespräch über den Ausgang der Provokation und die sich ergebenden Konsequenzen indiziert. Bei negativem Testergebnis sollte der Patient aufgefordert werden, die provozierten Nahrungsmittel nun in die Ernährung

- Tab. 7 Indikationen für die verschiedenen Formen einer Nahrungsmittelprovokation [14].

\begin{tabular}{l|l|}
\hline \begin{tabular}{l} 
Ausgangspunkt \\
\hline klare kürzliche Anaphylaxieanamnese $+\mathrm{ggE}$
\end{tabular} & Provokationstest \\
\hline \begin{tabular}{l} 
fragliche Anaphylaxieanamnese $\pm \mathrm{lgE}$ \\
\hline klares OAS + IgE
\end{tabular} & keine Provokation, strenge Diät \\
\hline keine Besserung unter Elimination bei Ekzem oder unspezifischen Symptomen & keine Provokation, eingeschränkte Diät \\
\hline Einführung des NM bei hoch sensibilisierten Patienten ohne bisherige Exposition & keine Provokation, keine Diät \\
\hline Re-Provokation nach langer Elimination & DBPCFC oder offen \\
\hline Verdacht auf isolierte Spätreaktionen bei Ekzem & DBPCFC oder offen \\
\hline Toleranzüberprüfung bei FPIES & DBPCFC \\
\hline Toleranzüberprüfung bei allergischer Proktokolitis & offene Provokation \\
\hline ängstlicher Patient/Eltern mit IgE-vermittelter Allergie & offene Provokation \\
\hline subjektive/atypische Symptome & DBPCFC \\
\hline $\begin{array}{l}\text { DBPCFC: Double-Blind Placebo-Controlled Food Challenge (doppelblind durchgeführter plazebokontrollierter Nahrungsmittel-Provokationstest); } \\
\text { FPIES: Food-Protein-Induced Enterocolitis-Syndrom; NM: Nahrungsmittel; OAS: orales Allergiesyndrom. }\end{array}$ \\
\hline
\end{tabular}


aufzunehmen und diese auch regelmäßig, d. h. mehrmals in der Woche, zu konsumieren. Durch eine nachfolgende Meidung könnte ansonsten die zum Zeitpunkt der Provokation bestehende Toleranz erneut verloren gehen.

\section{Merke}

Ist eine Allergie gegen das getestete Nahrungsmittel nachgewiesen worden, sollte der Patient auf die Notwendigkeit einer strikten Karenz hingewiesen werden.

Hierfür ist auch eine Aufklärung über die Deklarationsrichtlinien verpackter Lebensmittel erforderlich. Handelt es sich um ein essenzielles Nahrungsmittel, müssen Alternativen besprochen werden, um eine Mangelernährung zu vermeiden. Die Beratung wird daher am besten von einer Ernährungsfachkraft durchgeführt. Es ist sinnvoll, den Patienten auf Perspektiven der Toleranzentwicklung hinzuweisen und ggf. Re-Provokationen in adäquatem Abstand zu vereinbaren. Zusätzlich muss festgelegt werden, ob und welche Notfallmedikation vorzuhalten ist.

\section{PRAXIS}

Notfallset

Nach abgeschlossener Diagnostik muss der Patient hinsichtlich einer Notfallmedikation beraten werden. Besteht das Risiko, eine Anaphylaxie zu erleiden, sollten Bedarfsmedikamente in altersgerechter Dosis und Applikationsform verordnet und stets mitgeführt werden. Zu dem Set gehört zwingend ein Anaphylaxiepass, der genau beinhaltet, welche Medikation bei welcher Reaktion angewendet werden sollte. Das Notfallset sollte bei Anaphylaxie-gefährdeten Kindern ein Antihistaminikum, ein Steroid und einen Adrenalin-Autoinjektor enthalten. Bei begleitendem Asthma sollte zusätzlich Salbutamol bereitstehen.

\section{Therapie}

Trotz intensiver wissenschaftlicher Bemühungen haben sich bislang nur wenige praxistaugliche Therapieoptionen zur ursächlichen Behandlung der Nahrungsmittelallergie ergeben, und dann auch nur für die Erdnussallergie. Für die meisten Allergene bleibt es weiterhin das einzige mögliche Ziel, potenziell lebensbedrohliche Reaktionen durch konsequente Meidung des auslösenden Allergens zu verhindern.

\section{Allergenkarenz}

\section{Kinder mit Kuhmilch- oder Hühnerei-Allergie}

Die Auswahl der alternativen Therapienahrung für kuhmilchallergische Kinder ist altersabhängig. Im Säuglingsalter ist eine ernährungsphysiologisch vergleichbare Er-

\section{ZUSATZINFO}

\section{Allergenkennzeichnung}

Innerhalb der Europäischen Union wurden einheitliche und verbindliche Deklarationsrichtlinien formuliert, die für verpackte und lose Ware gelten. Es wurden 14 Allergene bzw. Allergengruppen definiert, die auf verpackten und beim Verkauf von losen Lebensmitteln in einem Zutatenverzeichnis deklariert werden müssen, wenn sie der Rezeptur absichtlich zugesetzt werden. Hierbei besteht die Kennzeichnungspflicht unabhängig von der zugesetzten Menge und betrifft auch Produkte, die aus den genannten Allergenen hergestellt werden, wie z. B. Fischgelatine oder Erdnussöl. Kennzeichnungspflichtige Nahrungsmittelallergene:

- Erdnuss

- Schalenfrüchte oder Nüsse (Haselnuss, Walnuss, Cashew, Pistazie, Paranuss, Mandel, Macadamianuss, Pekannuss)

- Soja

- Lupine

- Sesam

- Sellerie

- Senf

- glutenhaltige Getreide (Weizen, Roggen, Dinkel, Hafer, Gerste, Kamut)

- Hühnerei

- Milch inkl. Laktose (sämtliche Tiermilch)

- Fisch

- Krebstiere

- Weichtiere (z. B. Muscheln, Kopffüßler, Schnecken)

- Schwefeldioxid und Sulfite über $10 \mathrm{mg} / \mathrm{l}$

Die Allergene müssen im Zutatenverzeichnis besonders gekennzeichnet sein, z. B. durch Fettdruck oder Unterstreichung.

Nicht eindeutig gesetzlich geregelt ist die Deklaration unbeabsichtigter Kreuzkontaminationen bei der Herstellung, die sog. Spurenkennzeichnung. Hiermit werden Allergene angegeben, die nicht Teil der Rezeptur sind, aber aufgrund des Herstellungsprozesses in den Lebensmitteln zu finden sein können. Dies bedeutet zum einen, dass die Allergenmenge im Endprodukt nicht vorhersehbar ist, und zum anderen, dass der größte Teil der Produkte das Allergen nicht enthält. Viele Patienten gehen fälschlicherweise davon aus, dass in Produkten, auf denen Spuren gekennzeichnet sind, nur kleine Mengen des Allergens enthalten sind, und meiden diese Produkte daher nicht. Verschiedene Untersuchungen konnten jedoch zeigen, dass bis zu $10 \%$ der so deklarierten Nahrungsmittel das Allergen tatsächlich enthielten. Die hierbei gefundene Allergenmenge war keineswegs immer unbedeutend, sondern erreichte durchaus Bereiche, mit denen bei einem Teil der Patienten mit Reaktionen zu rechnen ist.

satznahrung notwendig. Zur Verfügung stehen Nahrungen auf der Basis eines extensiven Molken- oder Kaseinhydrolysats und Aminosäuremischungen. Bei den Extensivhydrolysaten sind die Kuhmilchproteine so weit aufgespalten, dass nur noch kurze Peptide enthalten sind. Etwa 95\% der lgE-vermittelten Kuhmilchallergiker reagie- 
ren auf diese Peptide nicht mehr. Aminosäuremischungen enthalten keine allergenen Proteine mehr. Sie sind vor allem dann sinnvoll, wenn die Kinder hochallergisch sind, und werden gern bei nicht-IgE-vermittelten Allergien eingesetzt.

\section{Cave}

Von den Therapienahrungen abzugrenzen sind die partiellen Hydrolysatnahrungen, die „HA-Nahrungen“. Hier ist der Hydrolysegrad der Kuhmilchproteine nicht ausreichend, um sicherzustellen, dass IgEAntikörper nicht mehr binden können. Sie sind daher als Ersatznahrung für kuhmilchallergische Kinder nicht geeignet.

Sojanahrung sollten nach Empfehlungen der Ernährungskommission der DGKJ [16] als Hauptnahrung erst nach dem 1. Geburtstag zum Einsatz kommen. Zuvor kann sie aber die Beikost ergänzen. Im 2. Lebensjahr stellt sie eine gute und preisgünstige sowie ernährungsphysiologisch sinnvolle Alternativnahrung dar. Vor dem erstmaligen Verzehr einer Sojanahrung bei einem kuhmilchallergischen Kind sollte durch gezielte Diagnostik (Prick-Test, IgE-Serologie) eine Sensibilisierung gegen Soja ausgeschlossen werden, auch wenn es keine Kreuzreaktionen gibt und Ko-Sensibilisierungen selten sind.

Weitere, häufig gewählte Nahrungsalternativen sind mit Kalzium angereicherte Reismilch oder Hafermilch. Nicht geeignet als Ersatznahrung sind hingegen andere Tiermilchsorten wie Ziegenmilch oder Schafsmilch, da das Kreuzreaktivitätsrisiko über $90 \%$ beträgt.

\section{Merke}

Da eine adäquate Nährstoffversorgung für nicht gestillte Säuglinge und Kleinkinder für das Wachstum und die Entwicklung von entscheidender Bedeutung ist, sollte die Indikation zu einer qualifizierten $\mathrm{Er}$ nährungsberatung großzügig gestellt werden.

Es ist bekannt, dass 60-70\% kuhmilchallergischer Patienten Kuhmilch in stark erhitzter Form tolerieren. Für die Toleranz ist ein hoher und lange dauernder Erhitzungsprozess des Milchprodukts notwendig, der die Proteine ausreichend denaturiert (z. B. Backen). Die regelmäßige Gabe stark erhitzter Produkte kann möglicherweise die Toleranzentwicklung fördern. Dies gilt auch für Hühnereiallergiker. Circa 70\% vertragen stark erhitztes Hühnerei z. B. in Backwaren.

\section{Kinder mit „Nuss“-Allergien}

In die Gruppe der Saaten, die umgangssprachlich als „Nüsse“ bezeichnet werden, fallen verschiedene Spezies, die nur eine begrenzte klinische, aber serologisch oft vorhandene Kreuzreaktivität aufweisen, und zwar Schalenfrüchte bzw. Nüsse, Erdnuss/Hülsenfrüchte und Ölsaaten wie Sesam oder Mohn. Da Erdnüsse zu den Hülsenfrüch- ten und nicht zu den Nüssen zählen, ist eine Reaktion auf Schalenfrüchte bei Erdnussallergikern nur in 20 bis maximal 50\% der Fälle zu erwarten. Eine vorsorgliche Meidung ist, insbesondere wenn schon eine Exposition stattgefunden hat, daher nicht sinnvoll. Ähnlich ist es bei Hülsenfrüchten. So sind bei Erdnussallergikern serologische Kreuzreaktionen gegen Soja und andere Hülsenfrüchte die Regel, wohingegen eine klinisch relevante Allergie nur selten auftritt.

Die Gruppe der Schalenfrüchte schließt eng verwandte Allergene ein, z. B. Pekan- und Walnuss (Nüsse) sowie Pistazie und Cashew (Steinfrüchte). Hier ist eine Kreuzallergie gegen das jeweils andere Allergen mit hoher Sicherheit zu erwarten. Die Wahrscheinlichkeit einer Allergie gegen mehrere Vertreter der Schalenfrüchte steigt bis zum Jugendlichenalter an, in dem bis zu 50\% der Patienten von einer Kreuzallergie gegen verschiedene Schalenfrüchte betroffen sind. Trotzdem sollten bereits vertragene Schalenfrüchte bei einer Allergie gegen eine einzelne andere Schalenfrucht nicht gemieden werden.

\section{Kinder mit atopischem Ekzem}

Während sich für Patienten mit Nahrungsmittelanaphylaxie keine Alternative zu einer konsequenten Diät ergibt, sollte bei Patienten mit atopischem Ekzem der Nutzen einer Karenzdiät dem Aufwand der Ekzem-Lokaltherapie gegenübergestellt werden. Bei Kindern mit leichtem bis mäßig schwerem atopischem Ekzem, das mit Basismaßnahmen und ohne regelmäßige antiinflammatorische Lokaltherapie gut zu kontrollieren ist, kann eine Diät aufwendiger und für die Lebensqualität beeinträchtigender sein als eine Basis-Externatherapie.

Bei voll gestillten Kindern mit atopischem Ekzem sollte die Relevanz einer nachgewiesenen Sensibilisierung durch eine diagnostische Diät und anschließende Re-Exposition der Mutter überprüft werden. Alle auf diese Art und Weise in ihrer Relevanz bestätigten Nahrungsmittelallergene sollten von der Mutter konsequent gemieden werden. Die Weitergabe von intakten Nahrungsmittelallergenen über die Muttermilch ist individuell unterschiedlich. Löst die Fütterung von Muttermilch bei einem allergischen Kind keine Symptome aus, obwohl die Mutter das Nahrungsmittel konsumiert, muss sie keine Diät einhalten. Da eine ausreichende Kalziumzufuhr für die stillende Mutter essenziell ist, sollte die Mutter eine Ernährungsberatung erhalten.

\section{Immuntherapie}

In den letzten Jahren wurden verstärkte Anstrengungen unternommen, eine Immuntherapie für Patienten mit Nahrungsmittelallergien zu implementieren. Prinzipiell werden verschiedene Methoden verfolgt, von denen 2 am vielversprechendsten sind: die orale und die epikutane Immuntherapie. Das primäre Ziel bei beiden Therapieformen ist, die individuelle Reaktionsschwelle des Patien- 
ten anzuheben, damit Reaktionen bei versehentlichem Verzehr unwahrscheinlich werden. Eine komplette Toleranz, das heißt der ungefährdete unregelmäßige Konsum, lässt sich meist nicht erreichen. Bei einigen Patienten ist aber eine Desensibilisierung möglich. Hier ist ein weitgehend unbeschränkter Konsum des Allergens möglich, solange der Patient das Allergen täglich zu sich nimmt. Pausiert er die Einnahme, geht die Verträglichkeit verloren.

\section{Orale Immuntherapie}

Bei der oralen Immuntherapie wird dem Patienten das auslösende Nahrungsmittelallergen in aufsteigender Menge täglich einmal oral verabreicht. Die Aufdosierungsphase bis zur angestrebten Zieldosis nimmt oft mehrere Monate in Anspruch. In Studien zur Therapie der Erdnussallergie mit einem kommerziellen Extrakt aus standardisiertem Erdnussmehl konnte die Mehrzahl der Patienten eine teils deutliche Anhebung der Reaktionsschwelle erreichen [17]. Auch reagieren die Patienten in der Provokation nach Therapie mit schwächeren Symptomen. Eine dauerhafte Toleranz wird allerdings nur bei wenigen Patienten erreicht. Ein entscheidendes Problem der Therapie ist die Rate an Nebenwirkungen. Diese sind zumeist mild und häufig gastrointestinal wie Bauchschmerzen und Übelkeit oder kutan mit Urtikaria oder Juckreiz. In einer großen Metaanalyse zeigte sich aber auch, dass die Patienten unter oraler Immuntherapie im 1. Therapiejahr deutlich häufiger eine Anaphylaxie erleben und mehr Adrenalingaben benötigen als die Patienten in der Placebogruppe [18].

\section{Merke}

Eine kommerzielle Therapie wird bislang nur für Erdnussallergie erprobt. Es existieren noch keine standardisierten Therapieprotokolle zu routinemäßigen Anwendungen bei anderen Allergenen.

\section{Epikutane Immuntherapie}

Bei der epikutanen Immuntherapie wird ein mit kleinsten Mengen eines Allergens beschichteter Film mit einem speziellen Pflaster auf die Haut aufgebracht. Durch die Verdunstung der Haut löst sich das Allergen von dem Film und wird von der Haut aufgenommen. Dort wird es prozessiert und entfaltet eine tolerogene Wirkung. Bei dieser Therapie treten kaum systemische Nebenwirkungen auf, und es wurde nur vereinzelt über Anaphylaxien berichtet. Die Mehrzahl der Patienten entwickelt lokale Nebenwirkungen der Haut am Ort der Applikation. Auch hier wird nach einer 3-jährigen Therapie bei der Mehrzahl der Patienten eine Anhebung der Reaktionsschwelle erreicht, jedoch in geringerem Ausmaß im Vergleich zur oralen Immuntherapie [19].
Merke

Für Patienten mit pollenassoziierten Nahrungsmittelallergien existieren noch keine etablierten Therapien. Eine spezifische Immuntherapie zur Behandlung der auslösenden Pollenallergie verbessert die Beschwerden nur bei rund der Hälfte der Patienten.

\section{Konsum stark erhitzter Produkte}

Bei der Gabe von stark erhitzter Milch oder stark erhitztem Hühnerei bei kuhmilch- oder hühnereiallergischen Kindern handelt es sich vermutlich nicht um eine Toleranzinduktion im eigentlichen Sinne. Dennoch haben sich klare Hinweise darauf ergeben, dass durch den regelmäßigen Konsum dieser stark erhitzten Produkte eine Toleranzentwicklung gegenüber Kuhmilch bzw. Hühnerei beschleunigt wird.

\section{Probiotika}

Abgesehen von den Verfahren zur Immuntherapie existieren außerhalb von Akutsituationen keine sinnvollen medikamentösen Therapien IgE-vermittelter Nahrungsmittelallergien. Die Supplementation von Probiotika zur Nahrung bei Kuhmilchallergikern wird derzeit noch kontrovers diskutiert. In einigen Studien, die bislang noch nicht repliziert wurden, konnte durch die Zugabe von Lactobacillus GG zu einer extensiv hydrolysierten Kuhmilch-Formula die Toleranzentwicklung besonders bei nicht-IgE-vermittelten Allergien beschleunigt werden. Mit anderen Pro- oder Synbiotika konnte dies am Menschen noch nicht gezeigt werden. Insgesamt ist daher die Datenlage noch zu schwach für eine generelle Empfehlung.

\section{Prognose}

Die Prognose einer Nahrungsmittelallergie hängt sowohl vom auslösenden Allergen als auch vom vorherrschenden Pathomechanismus ab. Nicht-IgE-vermittelte Allergien des Säuglings- und Kleinkindalters haben eine sehr gute Prognose. So ist bei der allergischen Proktokolitis des Säuglings meist nach 1-2 Jahren eine spontane Toleranzentwicklung zu erwarten. Auch für das FPIES wird beschrieben, dass sich in mehr als $50 \%$ der Fälle bis zum 3. Lebensjahr eine Symptomfreiheit einstellt. Allerdings hängt dies vom primär auslösenden Allergen ab. Bei der eosinophilen Ösophagitis ist die Langzeitprognose unklar. Es wird aber davon ausgegangen, dass betroffene Patienten einen chronischen Krankheitsverlauf zeigen.

Bei den IgE-vermittelten Allergien hängt die Prognose vor allem vom auslösenden Nahrungsmittel ab. Die Gesamtprognose der IgE-vermittelten Kuhmilchallergie ist gut, da die Mehrheit der Patienten bis zum Erreichen des Schulalters eine Toleranz entwickelt. Ähnlich gut ist die Prognose der Weizen- und, etwas schlechter, der Hühnereiallergie. Hingegen ist bei Allergien gegen Erdnuss, Schalenfrüchte und Fisch mit einer Persistenz der Allergie 
FALLBEISPIEL

\section{Alexander - Teil 4}

Aufgrund der deutlich gesunkenen IgE-Werte gegen Ara h 2 bei der lgE-Bestimmung mit 5 Jahren wird be Alexander vor der Einschulung eine offene ErdnussProvokation durchgeführt. Diese fällt negativ aus. Die Eltern erhalten die Empfehlung, Erdnuss nun an mindestens 3 Tagen die Woche zu verabreichen.

zu rechnen. Nur ein kleiner Teil der Patienten, bei Erdnuss z. B. bis zu $20 \%$ wird im Laufe der Zeit tolerant. Dies betrifft vor allem die Kinder im Vorschulalter.

Generell ist für die Abschätzung der Prognose die Höhe des spezifischen lgE-Werts relevant. Je höher der jemals gemessene Wert war, desto länger persistiert die Allergie. Ein weiterer Anhaltspunkt zur Vorhersage einer eingetretenen Toleranz ist der Verlauf der IgE-Diagnostik. Sowohl eine Abnahme der Reaktivität im Prick-Test als auch des spezifischen lgE können als Hinweis auf eine mögliche Toleranzentwicklung gewertet werden.

\section{KERNAUSSAGEN}

- Nahrungsmittelallergien werden von Patienten und deren Eltern sehr häufig als Auslöser verschiedenster Beschwerden vermutet, nach sinnvoller und gründlicher Diagnostik lässt sich im größten Teil der Fälle eine Allergie jedoch ausschließen.

- Es werden nicht-IgE-vermittelte Allergien von IgE-vermittelten Allergien unterschieden. Das Symptomspektrum ist groß und reicht von allergischen Sofortreaktionen über Ekzemauslösung und -verstärkung bis hin zu verschiedenen gastrointestinalen Beschwerden.

- Die Diagnostik besteht aus der Anamnese und dem Nachweis einer Sensibilisierung mittels Prick-Test oder Bestimmung spezifischer IgE-Antikörper. Zur endgültigen Klärung der Relevanz einer Sensibilisierung ist oft eine Nahrungsmittelprovokation notwendig.

- Die einzige zurzeit empfehlenswerte Therapie stellt die konsequente Allergenkarenz und das Mitführen einer Notfallapotheke dar.

- Moderne Immuntherapien zur Behandlung der Erdnussallergie sind in Entwicklung und werden in Kürze Marktreife erreichen.

- Die Prognose der Nahrungsmittelallergie ist gut. Bei Allergien gegen Grundnahrungsmittel ist in der Regel bis zum Schulalter eine Toleranz eingetreten.

\section{Interessenkonflikt}

\section{Erklärung zu finanziellen Interessen}

Forschungsförderung erhalten: nein; Honorar/geldwerten Vorteil für Referententätigkeit erhalten: ja, von einer anderen Institution (Pharma- oder Medizintechnikfirma usw.); Bezahlter Berater/interner Schulungsreferent/Gehaltsempfänger: ja, von einer anderen Institution (Pharma- oder Medizintechnikfirma usw.); Patent/Geschäftsanteile/Aktien (Autor/Partner, Ehepartner, Kinder) an im Bereich der Medizin aktiven Firma: nein; Patent/Geschäftsanteile/Aktien (Autor/Partner, Ehepartner, Kinder) an zu Sponsoren dieser Fortbildung bzw. durch die Fortbildung in ihren Geschäftsinteressen berührten Firma: nein.

Erklärung zu nichtfinanziellen Interessen

Gesellschaft für Pädiatrische Allergologie e. V., 2. Vorsitzender; Westdeutsche Arbeitsgemeinschaft für Pädiatrische Pneumologie und Allergologie e.V., 1. Vorsitzender; Arbeitsgemeinschaft Anaphylaxie Training und Edukation e.V., Vorstand.

\section{Autorinnen/Autoren}

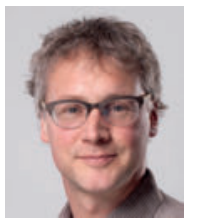

\section{Lars Lange}

Dr. med., geb. 1970, Studium der Humanmedizin an der Universität zu Köln, 1998-2007 Ausbildung zum Facharzt für Kinder- und Jugendmedizin, Allergologie und Kinder-Pneumologie an der Kinderklinik der Universität zu Köln. Seit 2008 Oberarzt und Chefarztvertreter in der Abteilung für Kinder- und Jugendmedizin am St. MarienHospital, Bonn. Forschungsschwerpunkte: Therapie und Diagnostik der Nahrungsmittelallergie bei Kindern und Jugendlichen, Schulungsprogramme für Anaphylaxie-kranke Kinder und Jugendliche, Medikamentenallergien, atopische Dermatitis.

Korrespondenzadresse

Dr. med. Lars Lange

GFO-Kliniken, Bonn. St. Marien-Hospital

Robert-Koch-Straße 1

53115 Bonn

Deutschland

Lars.Lange@gfo-kliniken-bonn.de

Wissenschaftlich verantwortlich gemäß Zertifizierungsbestimmungen

Wissenschaftlich verantwortlich gemäß Zertifizierungsbestimmungen für diesen Beitrag ist Dr. med. Lars Lange, Bonn. 
[1] Grabenhenrich L, Trendelenburg V, Bellach J et al. Frequency of food allergy in school-aged children in eight European countries - The EuroPrevall-iFAAM birth cohort. Allergy 2020; 75: 2294-2308

[2] Schoemaker AA, Sprikkelman AB, Grimshaw KE et al. Incidence and natural history of challenge-proven cow's milk allergy in European children - EuroPrevall birth cohort. Allergy 2015; 70: 963-972

[3] Lack G. Update on risk factors for food allergy. J Allergy Clin Immunol 2012; 129: 1187-1197

[4] Du Toit G, Roberts G, Sayre PH et al. Randomized trial of peanut consumption in infants at risk for peanut allergy. $\mathrm{N}$ Engl J Med 2015; 372: 803-813

[5] Worm M, Reese I, Ballmer-Weber B et al. Leitlinie zum Management IgE-vermittelter Nahrungsmittelallergien. Allergo J Int 2015; 24: 256-293

[6] Nowak-Wegrzyn A, Chehade M, Groetch M et al. International consensus guidelines for the diagnosis and management of food protein enterocolitis syndrome: Executive summaryworkgroup report of the adverse reactions to foods committee. J Allergy Clin Immunol 2017; 139: 1111-1126

[7] Lange L. Allergologische Diagnostik bei atopischem Ekzem im Kindesalter: wann sinnvoll, wann überflüssig? Pädiatrische Allergologie 2017; Sonderheft Neurodermitis: 17-24

[8] Gernert S, Arens A, Lange L. Diagnostik bei Nahrungsmittelallergien. Päd Allergologie 2019; Sonderheft Nahrungsmittelallergien: 21-30

[9] Beyer K, Grabenhenrich L, Härtl M et al. Predictive values of component-specific IgE for the outcome of peanut and hazelnut food challenges in children. Allergy 2015; 70: 90-98

[10] Ballmer-Weber BK, Lidholm J, Lange L et al. Allergen Recognition Patterns in Walnut Allergy Are Age Dependent and Correlate with the Severity of Allergic Reactions. J Allergy Clin Immunol Pract 2019; 7: 1560-1567

[11] Lange L, Lasota L, Finger A et al. Ana o 3-specific IgE is a good predictor for clinically relevant cashew allergy in children. Allergy 2017; 72: 598-603
[12] Kleine-Tebbe J, Reese I, Ballmer-Weber B et al. Keine Empfehlung für IgG und IgG4-Bestimmung gegen Nahrungsmittel. Allergo J 2009; 18: 267-268

[13] Lange L, Beyer K für die AG Nahrungsmittelallergie der GPA. Orale Nahrungsmittelprovokationen bei Verdacht auf eine Nahrungsmittelallergie im Säuglings- und Kindesalter. Pädiatrische Allergologie 2019; Sonderheft Nahrungsmittelallergie: $32-48$

[14] Niggemann B, Rolinck-Werninghaus C, Mehl A et al. Controlled oral food challenges in children - when indicated, when superfluous? Allergy 2005; 60: 865-870

[15] Flinterman AE, Knulst AC, Meijer $Y$ et al. Acute allergic reactions in children with AEDS after prolonged cow's milk elimination diets. Allergy 2006; 61: 370-374

[16] Ernährungskommission der Deutschen Gesellschaft für Kinder- und Jugendmedizin, Ernährungskommission der Schweizerischen Gesellschaft für Pädiatrie. Stellungnahme zur Verwendung von Säuglingsnahrungen auf Sojaeiweißbasis. Monatsschr Kinderheilkd 2006; 154: 913-916

[17] Vickery BP, Vereda A, Casale TB et al. the PALISADE group of investigators. AR101 Oral Immunotherapy for Peanut Allergy. N Engl J Med 2018; 379: 1991-2001

[18] Chu DK, Wood RA, French $S$ et al. Oral immunotherapy for peanut allergy (PACE): a systematic review and meta-analysis of efficacy and safety. Lancet 2019; 393: 2222-2232

[19] Fleischer DM, Greenhawt M, Sussman G et al. Effect of Epicutaneous Immunotherapy vs. Placebo on Reaction to Peanut Protein Ingestion Among Children With Peanut Allergy: The PEPITES Randomized Clinical Trial. JAMA 2019; 321: 446-455

\section{Bibliografie}

Pädiatrie up2date 2020; 15: 343-359

DOI $10.1055 / \mathrm{a}-0892-5839$

ISSN 1611-6445

(c) 2020. Thieme. All rights reserved.

Georg Thieme Verlag KG, Rüdigerstraße 14,

70469 Stuttgart, Germany 


\section{Punkte sammeln auf CME.thieme.de}

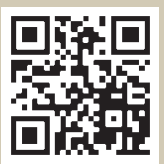

Diese Fortbildungseinheit ist in der Regel 12 Monate online für die Teilnahme verfügbar.

Den genauen Einsendeschluss finden Sie unter https://cme.thieme.de.

Sollten Sie Fragen zur Online-Teilnahme haben, finden Sie unter https://cme.thieme.de/hilfe

eine ausführliche Anleitung. Wir wünschen viel Erfolg beim Beantworten

der Fragen!

Unter https://eref.thieme.de/CXCY5CS oder über den QR-Code kommen Sie direkt zur Startseite des Wissenstests.

VNR 2760512020158720173

\section{Frage 1}

Welche Aussage zur Häufigkeit der Nahrungsmittelallergie ist richtig?

A Nahrungsmittelallergien bestehen bei ca. 16\% der deutschen Kinder und Jugendlichen.

B Eines der häufigsten Nahrungsmittelallergene in Deutschland ist Kuhmilch.

C Die Rate an Allergien gegen Hühnerei ist im Jugendlichenalter am höchsten.

D Die meisten allergischen Reaktionen auf Kuhmilch verlaufen unter dem Bild einer allergischen Proktokolitis.

E Die Rate an Nahrungsmittelallergien ist in Europa vergleichbar.

\section{Frage 2}

Welche Aussage zur Pathogenese von Nahrungsmittelallergien ist falsch?

A IgE-vermittelte Nahrungsmittelsensibilisierungen entstehen häufig auf epikutanem Weg.

B Die diskontinuierliche Gabe eines potenten Allergens im 1. Lebensjahr kann eine Allergieentstehung fördern.

C Die pathogenetischen Mechanismen, die zu einer nicht-IgEvermittelten Nahrungsmittelallergie führen, sind weitgehend verstanden.

D Eine defekte Hautbarriere prädisponiert zu einer Nahrungsmittelallergie, unabhängig vom Vorliegen einer atopischen Dermatitis.

E Potenziell allergene Nahrungsmittel sollten früh in die Ernährung von Säuglingen eingeführt werden, besonders bei Risikokindern.

\section{Frage 3}

Spezifisches IgE gegen welche Allergenkomponenten ist zur Vorhersage eines Anaphylaxierisikos nicht geeignet?
A Ara h 2 aus Erdnuss
B Jug $r 1$ aus Walnuss
C Cor a 14 aus Haselnuss
D Cor a 1 aus Haselnuss
E Ana o 3 aus Cashew

\section{Frage 4}

Welches Nahrungsmittel ist für einen nativen Prick-zu-Prick-Test geeignet?
A Senf
B Tomate
C Erdnuss
D Erdbeere
E Kiwi

\section{Frage 5}

Zu den Diagnosekriterien für ein akutes FPIES gehört welches klinische Zeichen?
A blutige Diarrhö
B sofort auftretendes Erbrechen nach Allergenaufnahme
C akute Urtikaria
D erstmaliges Auftreten im 1. Lebensjahr
E wiederholtes Erbrechen 1-4 Stunden nach Allergenaufnah- me

\section{Frage 6}

Welche Aussage zu pollenassoziierten Nahrungsmittelallergien trifft zu?

A Zur vorbeugenden Diagnostik sollte spezifisches IgE gegen alle potenziell kreuzreagierenden Nahrungsmittel bestimmt werden.

B Zur Diagnostik reichen oft die Anamnese und die Bestimmung des IgE gegen die Leitallergene der auslösenden Pollen aus.

C Bei nachgewiesener Allergie ist eine konsequente Meidung des Nahrungsmittels erforderlich.

D Eine bedrohliche Obstruktion der oberen Atemwege tritt häufig auf.

E Eine pollenassoziierte Nahrungsmittelallergie tritt besonders häufig bei schwerer Rhinokonjunktivitis auf.

\section{- Weitere Fragen auf der folgenden Seite...}




\section{Punkte samneln auf CME.thieme.de}

\section{Fortsetzung ...}

\section{Frage 7}

Welche Erkrankung ist keine Indikation für eine doppelblinde, plazebokontrollierte Nahrungsmittelallergie?

A Nachweis einer pollenassoziierten Nahrungsmittelallergie

B Verdacht auf isolierte Spätreaktion bei atopischem Ekzem

C Einführung eines nie konsumierten Nahrungsmittels bei vorhandener Sensibilisierung

D Überprüfung, ob eine Toleranz nach Karenz eingetreten ist

E Verdacht auf isoliert auftretende Bauchschmerzen nach einem bestimmten Nahrungsmittel

\section{Frage 8}

Was zählt nicht zu den deklarationspflichtigen Nahrungsmittelallergenen?
A Erdnuss
B Pekannuss
C Pinienkerne
D Lupine
E Kamut

\section{Frage 9}

Welche Aussage zur Immuntherapie mit Nahrungmittelallergenen trifft zu?

A Eine kommerzielle Therapie steht bereits seit Anfang 2020 zur Verfügung.

B Die meisten Patienten können nach Abschluss der Therapie Erdnuss in beliebiger Menge konsumieren.

C Durch die orale Immuntherapie steigt die Häufigkeit von Anaphylaxien im 1. Therapiejahr.

D Die epikutane Immuntherapie wird in der Regel nach 1 Jahr beendet.

E Zurzeit sind kommerzielle Immuntherapien für Hühnerei und Erdnuss in der Erprobung.

\section{Frage 10}

Was ist kein positiver Prognosefaktor der Kuhmilchallergie?

A kleine Prick-Test-Quaddel auf Kuhmilch bei Erkrankungsbeginn

B niedriges spezifisches IgE bei Erkrankungsbeginn

C abfallende Quaddelgröße im Prick-Test

D allergische Proktokolitis als Erkrankung

E eosinophile Ösophagitis als Erkrankung 